\title{
Author Index
}

Abdel-wahab, Z.A., Gillanders, W.E., Darrow, T.L., and Seigler, H.F. Generation of human IgG, $\operatorname{IgA}$, and IgM anti-melanoma monoclonal antibodies utilizing lymphocytes of an actively immunized melanoma patient, 32

Abercrombie, D.: See Knight, D.M., 129

Agha, R.: See Gupta, K.C., 25

Allen, L.: See Saleh, M.N., 19

Amin, A.R.: See Shin, S.-U., 65

Arevalo-Moore, M.: See Looney, J.E., 191

Aw, D.: See Shepherd, A.J., 168

Bieber, M.: See Glasky, M.S., 114

Boyd, J.E.: See Shepherd, A.J., 168

Brodeur, B.R.: See Gupta, K.C., 25

Brown, K.: See Horgan, C., 153

Burlingame, R.W. and Rubin, R.L. Antihistone autoantibodies recognize centromeric heterochromatin in metaphase chromosomes and hidden eiptopes in interphase cells, 40

Buskila, D., Weigl, D., and Shoenfeld, Y. The detection of anti-Ro/SS-A and antiLa/SS-B activity of human serum monoclonal immunoglobulins (monoclonal gammopathies), 75

Chevaleyre, J.: See Jallu, V., 93 Coll, J.T.: See Lo, K.-M., 123

Crowe, J.S.: See Lewis, A.P., 146

Daddona, P.E.: See Looney, J.E., 191 Dalesandro, M.R.: See Looney, J.E., 191 Darrow, T.L.: See Abdel-wahab, Z.A., 32 Daugherty, B.L.: See Spatz, L.A., 107 de Groot, C.: See Kwekkeboom, J., 48 DeMartino, J.A.: See Spatz, L.A., 107 Döcke, W.: See Seifert, M., 86

Ehrlich, P.H., Moustafa, Z.A., Justice, J.C., Harfeldt, K.E., Kelley, R.L., and Östberg, L. Characterization of human monoclonal antibodies directed against hepatitis B surface antigen, 2

Epplen, C.: See Hampe, J., 186

Epplen, J.T.: See Hampe, J., 186

Foley, S.F.: See Lo, K.-M., 123

Gershwin, M.E.: See Naiki, M., 60 Gessner, P.: See Zimmermann, U., 14

Ghrayeb, J.: See Knight, D.M., 129; Looney, J.E., 191

Gillanders, W.E.: See Abdel-wahab, Z.A., 32

Gillies, S.D.: See Lo, K.-M., 123; Saleh, M.N., 19

Glasky, M.S., Yin, A., Smith, L.H., Bieber, M., and Teng, N.N.H. Adenocarcinoma-reactive human monoclonal antibody MS2B6 defines an antigen in simple glandular epithelium, 114

Grizzle, W.: See Saleh, M.N., 19

Grunow, R.: See Hampe, J., 186

Gupta, K.C., Agha, R., Santos, E., and Brodeur, B.R. Isolation of human monoclonal antibodies binding to $\mathrm{B}$ fragment of diphtheria toxin, 25
Hampe, J., Nürnberg, P., Epplen, C., Jahn, S., Grunow, R., and Epplen, J.T. Oligonucleotide fingerprinting as a means to identify and survey long-term cultured B cell hybridomas and $\mathrm{T}$ cell lines, 186

Harfeldt, K.E.: See Ehrlich, P.H., 2

Hay, B.N., Sorge, J.A., and Shopes, B. Bacteriophage cloning and Escherichia coli expression of a human IgG Fab, 81

Healey, M.C.: See Torres, A.R., 206

Henri, E.: See Jonak, Z.L., 177

Hogg, C.L.: See Shepherd, A.J., 168

Horgan, C., Brown, K., and Pincus, S.H. Variable region differences affect antibody binding to immobilized but not soluble antigen, 153

Jahn, S.: See Hampe, J., 186; Seifert, M., 86

Jallu, V., Pico, M., Chevaleyre, J., Vezon, G., Kunicki, T.J., and Nurden, A.T. Characterization of an antibody to the integrin $\beta 3$ subunit (GP IIIa) from a patient with neonatal thrombocytopenia and an inherited deficiency of GP IIbIIIa complexes in platelets (Glanzmann's thrombasthenia), 93

James, K.: See Shepherd, A.J., 168

Jensen, C.P.: See Jonak, Z.L., 177

Jochems, G.J., Klein, M.R., Zeijlemaker, W.P., and van Lier, R.A.W. Protein kinase $C$ regulates secretion of lymphotoxin (LT/TNF $\beta)$ and TNF $\alpha$ by human EBV-transformed B cells, 162

Johnston, A.V.: See Torres, A.R., 206

Jonak, Z.L., Henri, E., Trulli, S., Jensen, C.P., and Muirhead, K.A. Manipulation of human B cells to confer immortality, 177

Justice, J.C.: See Ehrlich, P.H., 2

Kawahara, H., Shirahata, S., Tachibana, H., and Murakami, H. In vitro immunization of human lymphocytes with human lung cancer cell line A549, 8

Kelley, R.L.: See Ehrlich, P.H., 2

Kenny, T.: See Naiki, M., 60'

Khazaeli, M.B.: See Saleh, M.N., 19

Klein, M.R.: See Jochems, G.J., 162

Klöck, G.: See Zimmermann, U., 14

Knight, D.M.: See Looney, J.E., 191

Knight, D.M., McDonough, M., Moore,

M.A., Abercrombie, D., Siegel, R., and Ghrayeb, J. Stable expression of cloned human antibody genes in murine myeloma cells, 129

Konishi, J.-E.: See Naiki, M., 60'

Kunicki, T.J.: See Jallu, V., 93

Kwekkeboom, J., de Groot, C., and Tager, J.M. Efficient electric fieldinduced generation of hybridomas from human B lymphocytes without prior activation in vitro, 48

Latov, N.: See Spatz, L.A., 107

Lewis, A.P., Parry, N., Peakman, T.C., and Crowe, J.S. Rescue and expression of human immunoglobulin genes to generate functional human monoclonal antibodies, 146
Lo, K.-M., Roy, A., Foley, S.F., Coll, J.T., and Gillies, S.D. Expression and secretion of an assembled tetrameric CH2-deleted antibody in E. coli, 123 LoBuglio, A.F.: See Saleh, M.N., 19

Looney, J.E., Knight, D.M., ArevaloMoore, M., Trinh, H., Pak, K.-Y., Dalesandro, M.R., Rieber, E.P., Reithmuller, G., Daddona, P.E., and Ghrayeb, J. High-level expression and characterization of a mouse-human chimeric CD4 antibody with therapeutic potential, 191

McDonough, M.: See Knight, D.M., 129

McKnight, M.E.: See Torres, A.R., 206

Mark, G.E.: See Spatz, L.A., 107

Moore, M.A.: See Knight, D.M., 129

Morrison, S.L.: See Shin, S.-U., 65

Moustafa, Z.A.: See Ehrlich, P.H., 2

Muirhead, K.A.: See Jonak, Z.L., 177

Murakami, H.: See Kawahara, H., 8

Naiki, M., Robbins, D.L., Kenny, T., Yago, H., Suehiro, S., Gershwin, M.E., and Konishi, J.-E. Characterization of monoclonal IgG antibodies produced by hybridomas derived from rheumatoid synovial cells, 60

Neil, G.A.: See Zimmermann, U., 14

Neil, G.A. and Sammons, D.W. Immunization of SCID-Hu mice and generation of anti-hepatitis B surface antigen-specific hybridomas by electrofusion, 201

Nurden, A.T.: See Jallu, V., 93

Nurnberg, P.: See Hampe, J., 186

Östberg, L.: See Ehrlich, P.H., 2

Pak, K.-Y.: See Looney, J.E., 191

Parren, P.W.H.I. Preparation of genetically engineered monoclonal antibodies for human immunotherapy, 137

Parry, N.: See Lewis, A.P., 146

Peakman, T.C.: See Lewis, A.P., 146

Pico, M.: See Jallu, V., 93

Pincus, S.H.: See Horgan, C., 153

Reisfeld, R.A.: See Saleh, M.N., 19

Rieber, E.P.: See Looney, J.E., 191

Riethmuller, G.: See Looney, J.E., 191

Robbins, D.L.: See Naiki, M., 60

Roy, A.: See Lo, K.-M., 123

Rubin, R.L.: See Burlingame, R.W., 40

Saleh, M.N., Khazaeli, M.B., Wheeler, R.H., Allen, L., Tilden, A.B., Grizzle, W., Reisfeld, R.A., Yu, A.L., Gillies, S.D., and LoBuglio, A.F. Phase I trial of the chimeric anti-GD2 monoclonal antibody chl 4.18 in patients with malignant melanoma, 19

Sammons, D.W.: See Zimmermann, U. 14

Sammons, W.: See Neil, G.A., 201

Santos, E.: See Gupta, K.C., 25

Schwab, J.: See Seifert, M., 86

Seifert, M., Jahn, S., Schwab, J., Döcke, W., Volk, H.-D., and von Baehr, R. 
Lymphocyte surface marker expression on hybridomas secreting human monoclonal antibodies, 86

Seigler, H.F.: See Abdel-wahab, Z.A., 32

Shepherd, A.J., Boyd, J.E., Hogg, C.L., Aw, D., and James, K. Susceptibility of human monoclonal antibody-producing $\mathrm{B}$ cell lines to infection by human immunodeficiency virus, 168

Shin, S.-U., Wei, C.-F., Amin, A.R., Thorbecke, G.J., and Morrison, S.L. Structural and functional properties of mouse-human chimeric IgD, 65

Shirahata, S.: See Kawahara, H., 8

Shoenfeld, Y.: See Buskila, D., 75

Shopes, B.: See Hay, B.N., 81

Siegel, R.: See Knight, D.M., 129

Smith, L.H.: See Glasky, M.S., 114

Sorge, J.A.: See Hay, B.N., 81
Spatz, L.A., Daugherty, B.L., DeMartino, J.A., Mark, G.E., and Latov, N. Expression of recombinant human antiMAG antibodies in nonlymphoid mammalian cells, 107

Suehiro, S.: See Naiki, M., 60'

Tachibana, H.: See Kawahara, H., 8

Tager, J.M.: See Kwekkeboom, J., 48

Teng, N.N.H.: See Glasky, M.S., 114

Thorbecke, G.J.: See Shin, S.-U., 65

Tilden, A.B.: See Saleh, M.N., 19

Torres, A.R., Healey, M.C., Johnston, A.V., and McKnight, M.E. Growth of hybridoma cells and antibody production in agamma calf serum, 206

Trinh, H.: See Looney, J.E., 191

Trulli, S.: See Jonak, Z.L., 177

van Lier, R.A.W.: See Jochems, G.J., 162
Vezon, G.: See Jallu, V., 93

Volk, H.-D.: See Seifert, M., 86 von Baehr, R.: See Seifert, M., 86

Wei, C.-F.: See Shin, S.-U., 65 Weigl, D.: See Buskila, D., 75 Wheeler, R.H.: See Saleh, M.N., 19

Yago, H.: See Naiki, M., 60' Yin, A.: See Glasky, M.S., 114 Yu, A.L.: See Saleh, M.N., 19

Zeijlemaker, W.P.: See Jochems, G.J., 162 Zimmerman, U., Klöck, G., Gessner, P., Sammons, D.W., and Neil, G.A. Microscale production of hybridomas by hypo-osmolar electrofusion, 14

\section{Subject Index}

\section{Affinity, 153}

Antibody, 81, 206

Antibody gene expression, 129

Antibody secretion, 65

Anti-GD2 antibody, 19

Anti-La/SS-B antibodies, 75

Anti-myelin associated glycoprotein, 107

Anti-Ro/SS-A antibodies, 75

Autoimmune diseases, 75

Bacterial expression, 123

B-cell, 162

B cell lines, 168

B fragment binding, 25

CD4, 191

cDNA cloning, 146

Cell culture, 186, 206

Cell surface marker expression, 86

CENP-A, 40

CH2-deleted antibody, 123

Chimeric 14.18, 19

Chimeric antibodies, 153

Chimeric antibody, 65

Chimeric monoclonal antibody, 191

Colon carcinoma, 114

Constitutive heterochromatin, 40

Diphtheria toxin, 25

DNA fingerprinting, 186

EBV, 162

EBV transformation, 186

Effector functions, 137

Electrofusion, 14, 48

Electroporation, 177

Expression, 146

Feeder cells, 186

Fusion frequency, 48
Gb-IIb-III complexes, 93

Gene expression, 81

Glanzmann's thrombasthenia, 93

Glycosylation, 65

HBsAg, 2

Hepatitis B, 2, 201

Histone $\mathrm{H} 3,40$

HIV-1, 168

HIV-2, 168

Human antibodies, 129

Human autoantibody, 93

Human B lymphocytes, 48

Human-human hybridomas, 8

Human hybridomas, 60, 201

Humanization, 137

Human lymphocytes, 25

Human monoclonal antibodies, 8, 25, 177

Human monoclonal antibody, 2, 114, 146, 168

Human-mouse hybridomas, 48

Hybridoma, 186, 206

Hybridoma cell lines, 86

Hybridoma production, 14

Hypo-osmolar, 14

$\operatorname{IgD}, 65$

Immobilized immune complexes, 153

Immortalization, 177

Immune-related thrombocytopenias, 93

Immunofluorescence, 40,86

Immunoglobulin, 81

Immunoglobulin rescue, 146

Immunotherapy, 137

In vitro immunization, 8, 25, 177

LT, 162

Lung cancer, 8
Malignant melanoma, 32

Metastatic melanoma, 19

Microchamber, 14

Monoclonal antibodies, 32

Monoclonal gammopathies, 75

Monoclonals, 206

M-T412, 191

Novel cell lines, 177

Nuclear matrix-intermediate filament, 114

Oncogenic DNA, 177

Ovarian carcinoma, 114

pelB leader, 123

PK-C, 162

Plasma cell dyscrasias, 75

Platelets, 93

Polymerase chain reaction, 81, 107

Recombinant antibodies, 129, 137

Rheumatoid arthritis, 60

Rheumatoid factors, 60

SCID-Hu mice, 201

Secretion, 123

Serum, 206

Serum-free, 206

Signal transduction, 162

Soluble immune complexes, 153

Specific active immunotherapy, 32

Subnucleosome, 40

TNF $\alpha, 162$

Toxin neutralization, 25

Tumor-associated antigen, 114

Variable genes, 107 